\title{
APLICAÇÃO DE DIFERENTES FONTES DE ENERGIA NA SÍNTESE ENZIMÁTICA DE BIODIESEL USANDO ÓLEO DE COCO BRUTO
}

\author{
M. V. S. OLIVEIRA ${ }^{1}$, P. C. M. DA ROS ${ }^{2}$, A. K. F. CARVALHO ${ }^{2}$, N. B. CARVALHO ${ }^{1}$, \\ A. T. FRICKS ${ }^{1}$, H. F. CASTRO ${ }^{2}$, C. M. F. SOARES ${ }^{1}$, A. S. LIMA ${ }^{1}$ \\ ${ }^{1}$ Universidade Tiradentes, ITP - Instituto de Tecnologia e Pesquisa, 300, Aracaju - SE, Brasil; \\ ${ }^{2}$ Escola de Engenharia de Lorena, USP, Depart. de Engenharia Química, Lorena - SP, Brasil. \\ E-mail para contato: oliveira.mvso@gmail.com
}

\begin{abstract}
RESUMO - O presente trabalho avaliou a eficiência de diferentes fontes de energia (aquecimento convencional, micro-ondas e ultrassom) na síntese enzimática de biodiesel a partir do óleo de coco bruto empregando etanol como agente acilante. As reações de transesterificação ocorreram a $40{ }^{\circ} \mathrm{C}$ empregando meios reacionais contendo óleo e etanol na razão molar óleo: etanol de 1:12 e utilizando como biocatalisadores Novozym ${ }^{\circledR}$ 435, lipase de Burkholderia cepacia imobilizada em diatomácea e imobilizada em suporte híbrido $\left(\mathrm{SiO}_{2}-\mathrm{PVA}\right)$. Nas reações submetidas ao aquecimento convencional foram obtidos rendimentos superiores a $98 \%$ para os três derivados imobilizados em tempos reacionais que variaram entre 24 a $72 \mathrm{~h}$. As reações assistidas por irradiação de micro-ondas alcançaram menores conversões em ésteres etílicos de 65-95\%, dependendo do derivado imobilizado. O melhor desempenho foi alcançado nas reações conduzidas em ultrassom que forneceram rendimentos elevados $(98 \%)$ com dois biocatalisadores (Burkholderia cepacia imobilizada em $\mathrm{SiO}_{2}$-PVA e Novozym 435) em 8 e 6h, respectivamente, reduzindo o tempo de reação estabelecido pelos processos que envolveram aquecimento convencional e micro-ondas.
\end{abstract}

\section{INTRODUÇÃO}

As reações de transesterificação de óleos vegetais por meio da aplicação de enzimas lipolíticas atualmente são investigadas e tem alcançado rendimentos significativos de ésteres etílicos (Stamenkovic et al., 2011). No processo, óleos residuais, como descrito por Oliveira et al. 2010 e Ribeiro et al., 2012 são utilizados como matéria-prima alternativa, a qual reduz a competição entre o uso de óleos para alimentos ou combustíveis, além de propiciar o menor custo do biocombustível sintetizado, eliminar subsídios financeiros à cadeia produtiva e minimizar a elevação dos preços de óleos comestíveis decorrente de sua menor demanda no mercado pela aplicação em síntese de biocombustível (Chen et al., 2012).

Outro ponto importante no processo de síntese de biodiesel é a aplicação de fontes de energia utilizadas no processo enzimático. Normalmente, as baixas taxas de reação do processo de transesterificação são oriundas da baixa transferência de massa do meio reacional no sistema imobilizado. No sentido de minimizar este efeito, vários métodos já foram estudados para intensificação do processo de produção de biodiesel, tais como ultrassom e micro-ondas. A energia da irradiação ultrassônica pode ativar vários mecanismos que podem afetar as reações químicas e processos de forma positiva, que não são sempre conhecidos, além do mais o uso de tecnologia de micro-ondas pode auxiliar o processo de transesterificação resultando em tempos de reação mais rápidos (Cintas et al., 2010; Kanitar et al., 2011; Veljkovic et al., 2012) .

Desta forma, o objetivo deste trabalho foi estudar comparativamente a aplicação de diferentes fontes de energia (aquecimento convencional, micro-ondas e ultrassom) na síntese de biodiesel a partir de óleo de coco bruto (sem pré-tratamento), utilizando lipases imobilizadas. 


\section{METODOLOGIA}

\subsection{Material}

Nas reações de transesterificação foram utilizados etanol anidro e óleo de coco bruto como fontes de matéria-prima. Algumas propriedades do óleo de coco utilizado neste trabalho estão apresentadas na Tabela 1. Os biocatalisadores utilizados foram: lipase de Burkholderia cepacia (BCL) imobilizada em $\mathrm{SiO}_{2}-\mathrm{PVA}$ $(1.805,5 \pm 137,7 \mathrm{U} / \mathrm{g}$ - processo de síntese e imobilização na seção 2.2) e preparações disponíveis comercialmente: lipase de Burkholderia cepacia imobilizada em diatomácea (3698,7 U/g) e Novozym 435.

Tabela 1 - Caracterização do óleo de coco bruto

\begin{tabular}{|c|c|}
\hline Densidade à $20^{\circ} \mathrm{C}$ & $0,92 \mathrm{~g} / \mathrm{cm}^{3}$ \\
\hline Massa Molecular & 701,79 \\
\hline Viscosidade Cinemática à $40{ }^{\circ} \mathrm{C}$ & $29 \mathrm{~mm}^{2} / \mathrm{s}$ \\
\hline Índice de Saponificação & $187 \mathrm{mg}_{\mathrm{KOH}} / \mathrm{g}$ \\
\hline Índice de Iodo & $18,50 \mathrm{gI}_{2} / 100 \mathrm{amostra}$ \\
\hline Índice de Acidez & $21,05 \mathrm{mg}_{\mathrm{KOH}} / \mathrm{g}$ \\
\hline Índice de Peróxido & $6,81 \mathrm{meq} / \mathrm{Kg}$ \\
\hline
\end{tabular}

\subsection{Síntese do Suporte ( $\mathrm{SiO}_{2}$-PVA) e Imobilização da Lipase}

$\mathrm{O}$ suporte $\mathrm{SiO}_{2}$-PVA foi preparado a partir da hidrólise e policondensação de tetraetoxissilano pela técnica sol-gel (Freitas et al., 2009). A imobilização da lipase de B. cepacia no suporte híbrido de $\mathrm{SiO}_{2}-\mathrm{PVA}$ ativado com epicloridrina foi realizada por ligação covalente de acordo com o método descrito por Da Rós et al. (2012). O suporte ativado foi embebido em hexano, sob agitação (100 rpm) durante $1 \mathrm{~h} \mathrm{a} 25^{\circ} \mathrm{C}$. Em seguida, o excesso de hexano foi removido e a lipase foi adicionada a uma razão de 1:4 grama de enzima por grama de suporte. PEG-1500 foi adicionado em conjunto com a solução de enzima a uma parte fixa (100 $\mu \mathrm{l} / \mathrm{g}$ de suporte). O sistema lipase-suporte foi mantido em contato durante $16 \mathrm{~h}$, a $4{ }^{\circ} \mathrm{C}$, em condições estáticas. Os derivados de lipase imobilizada foram filtrados (62HD membrana de nylon de Scheiz Seidengazefabrik AG, Thal Schweiz, Suíça) e cuidadosamente lavados com hexano.

\subsection{Síntese de Ésteres Etílicos}

Todos os ensaios foram realizados com $12 \mathrm{~g}$ de substrato, constituído por óleo de coco bruto e etanol anidro, sem a adição de solventes a $40^{\circ} \mathrm{C}$ e razão molar de $1: 12$ (condição estabelecida anteriormente - dados não mostrados). O meio reacional foi incubado com o biocatalisador numa proporção fixa em relação ao peso total dos reagentes. Para lipase de B. cepacia imobilizada em $\mathrm{SiO}_{2}$-PVA a proporção fixa foi de $20 \%$ (Freitas et al., 2009) enquanto que para biocatalisadores obtidos comercialmente (lipase de B. cepacia em diatomácea e Novozym 435) a proporção fixa correspondeu a $10 \%$. Para os estudos do perfil de conversão, uma alíquota do meio reacional foi retirada em vários intervalos de tempo para análise. O procedimento realizado para as diferentes fontes de energia são descritos a seguir:

Aquecimento convencional: os ensaios foram executados em reatores fechados com capacidade de 25 $\mathrm{mL}$, sob agitação magnética constante de $150 \mathrm{rpm}$ como relatado por Freitas et al. (2009). As reações foram realizadas por um período máximo de $72 \mathrm{~h}$. 
Micro-ondas: as reações foram realizadas em reator de micro-ondas (Modelo Discover/University -Wave Cem Corporation), o qual é composto por câmara interna cilíndrica de $75 \mathrm{~mm}$ de diâmetro e $100 \mathrm{~mm}$ de altura. O design cilíndrico garante irradiação homogênea do volume de trabalho que, por exemplo, está em falta nos fornos de micro-ondas convencionais disponíveis no mercado e, frequentemente utilizados em experiências, sem modificações. A potência máxima de operação foi de cerca de $300 \mathrm{~W}$. Um agitador magnético foi utilizado para promover a agitação do meio. A temperatura foi controlada, com o ar comprimido soprado para dentro da câmara. Este ar de refrigeração foi concebido para operar com diferentes níveis de pressão e, ou no modo de fluxo contínuo ou intermitente. A temperatura da reação foi monitorada por um sensor de infravermelho localizado na parte inferior da câmara. Um reator de vidro esférico de $100 \mathrm{~mL}$ foi acoplado a um condensador de refluxo para evitar a perda de etanol como de vapor (Da Rós et al. 2012).

Ultrassom: experimentos foram realizados em ultrassom UP 200S (Hielscher ultra-sônica Gmblt). Os processos no ultrassom operam em $200 \mathrm{~W}$ e frequência de $24 \mathrm{kHz}$. A amplitude e ciclo da reação foram reguladas para $30 \%$ e 0,7 , respectivamente e a temperatura foi mantida em torno de $54^{\circ} \mathrm{C}$. O sonotrodo de titânio S7 com um diâmetro de $7 \mathrm{~mm}$ e comprimento de $100 \mathrm{~mm}$ foi usado para transmitir as ondas ultrassônicas para dentro do líquido. Os experimentos foram realizados em reator de vidro esférico, com capacidade de $25 \mathrm{~mL}$.

\subsection{Determinações Analíticas}

Atividade enzimática: as atividades enzimáticas das amostras de lipase livre e imobilizadas foram determinados pelo método de emulsão de azeite de acordo com a alteração proposta de Soares et al. (1999). Uma unidade (U) de atividade enzimática foi definida como a quantidade de enzima que liberta 1 mol de ácidos graxos livres por min nas condições do ensaio $\left(37^{\circ} \mathrm{C}, \mathrm{pH} 7,0,5\right.$ minutos de incubação).

Análise dos ésteres etílicos: os ésteres etílicos formados foram analisados por cromatografia gasosa utilizando um modelo de Varian CG 3800 (Varian, Inc., Palo Alto, CA, EUA), equipado com detector de ionização de chama e 5\% DEGS CHR- WHP 80/100 de, 2,0 mm ID, em uma coluna empacotada de aço inoxidável (Restek, Frankel Comércio de Instrumentos Analíticos Ltd., SP, Brasil). Utilizou-se nitrogênio como gás de arraste com taxa de fluxo de $25 \mathrm{~mL} / \mathrm{min}$. A temperatura da coluna foi mantida a $90^{\circ} \mathrm{C}$ durante 3 min, aquecida a $120^{\circ} \mathrm{C}$ a $25^{\circ} \mathrm{C} / \mathrm{min}$ e manteve-se constante durante $10 \mathrm{~min}$. Em seguida, a temperatura foi programada a $25^{\circ} \mathrm{C} / \mathrm{min}$ até $170^{\circ} \mathrm{C}$ e manteve-se constante durante $15 \mathrm{~min}$. As temperaturas do injetor e do detector foram ajustadas a $250^{\circ} \mathrm{C}$. A coleta e análise dos dados foram realizadas utilizando o software Galaxie Chromatography Data System versão 1.9. As curvas de calibração foram construídas a partir de ésteres etílicos padrões usando hexanol como padrão interno. O rendimento de transesterificação foi calculado tomando por base a massa de teor de éster obtido por análise de GC e do éster teórico total de massa com base na razão molar da reação (Urioste et al., 2008).

\section{RESULTADOS E DISCUSSÃO 3.1. Aquecimento Convencional}

A conversão dos ácidos graxos presentes no óleo de coco bruto em ésteres etílicos com aquecimento convencional foram em torno de $98 \%$ com $72 \mathrm{~h}$ de reação para a lipase de B. cepacia imobilizada em $\mathrm{SiO}_{2^{-}}$ PVA e em diatomácea. Os dados obtidos para os sistemas imobilizados com a lipase de B. cepacia estão em concordância com a literatura que relatam capacidade na obtenção de conversões máximas de ésteres etílicos (Noureddini et al, 2005; Freitas et al, 2009). Uma conversão total, 100\%, foi obtida com o biocatalisador Novozym 435 provavelmente antes das 24h de reação. De acordo com Azócar et al. (2010), Novozym 435 é 
resistente e estável em comparação com outras lipases na presença de álcoois de cadeia curta. O perfil do rendimento de transesterificação em função do tempo para cada biocatalisador é ilustrado na Figura 1.

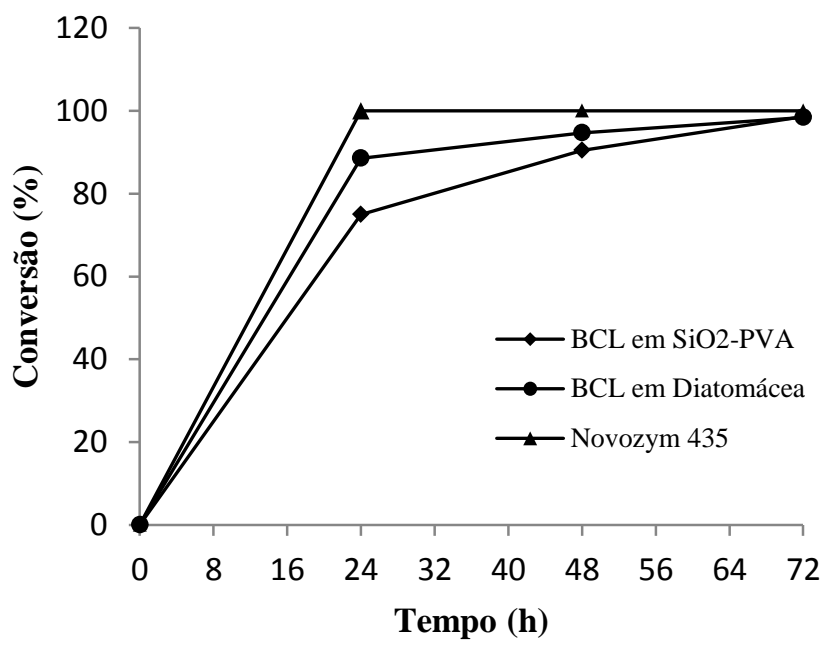

Figura 1 - Perfil da conversão em ésteres etílicos empregando aquecimento convencional.

\subsection{Micro-ondas}

A transesterificação em reator micro-ondas demonstrou conversões máximas de $65 \%$ para $B$. cepacia imobilizada em $\mathrm{SiO}_{2}$-PVA, 78,3\% para B. cepacia imobilizada em diatomácea e de aproximadamente $95 \%$ para Novozym 435, como pode ser visualizado na Figura 2. Estes resultados da transesterificação catalisada por sistemas imobilizados com a lipase de B. cepacia mostraram que a eficiência dos biocatalisadores foi inibida pela interação entre a radiação micro-ondas com as características do óleo de coco bruto utilizado como matéria-prima (acidez elevada: 21,05 $\mathrm{mgKOH} / \mathrm{g}$ e índice de peróxido: $6,81 \mathrm{meq} / \mathrm{Kg}$ ). De acordo com Ibrahim et al. (2007) o limite recomendado do índice de peróxido para uso de óleo como matéria-prima em reações enzimáticas é na ordem de $5 \mathrm{meq} / \mathrm{Kg}$.

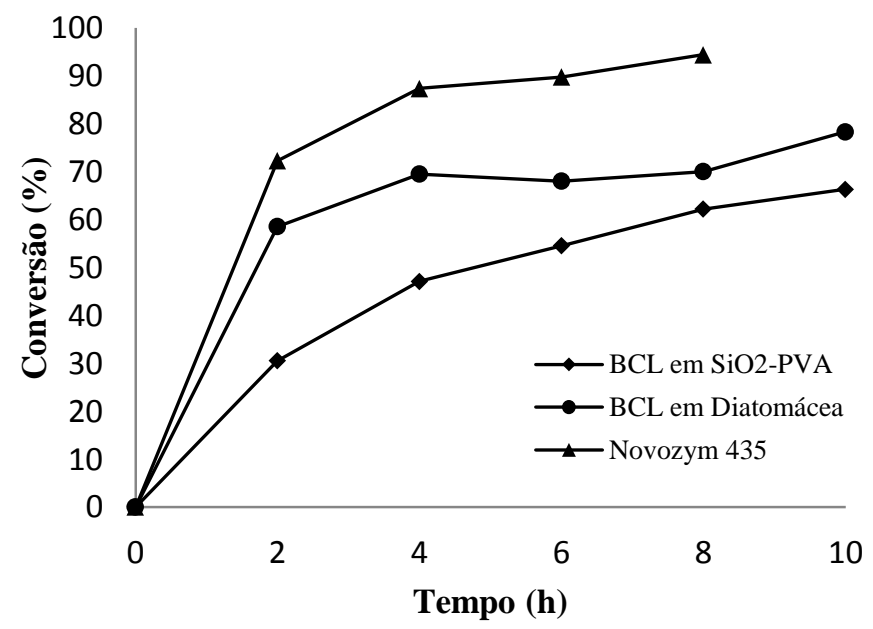

Figura 2 - Perfil da conversão em ésteres etílicos empregando micro-ondas. 


\subsection{Ultrassom}

O perfil da conversão do óleo de coco bruto empregando o ultrassom pode ser visualizado na Figura 3. Quando a reação foi catalisada por $B$. cepacia imobilizada em diatomácea a conversão permaneceu constante entre 4 e 6 h e atingiu a máxima conversão de 72,32\% para 8 h de reação. Este efeito observado ocorreu, provavelmente, devido à forma de imobilização da lipase e/ou do suporte utilizado, que interagiu negativamente com o fenômeno da cavitação levando a diminuição da estabilidade do biocatalisador. A reação de transesterificação utilizando B. cepacia imobilizada em $\mathrm{SiO}_{2}$-PVA atingiu $100 \%$ de conversão em ésteres etílicos no período de $8 \mathrm{~h}$, enquanto a Novozym 435 forneceu a mesma conversão em $6 \mathrm{~h}$. De acordo com Veljkovic et al. (2012), o emprego do ultrassom pode aumentar a atividade enzimática do biocatalisador Novozym 435 em reações de transesterificação.

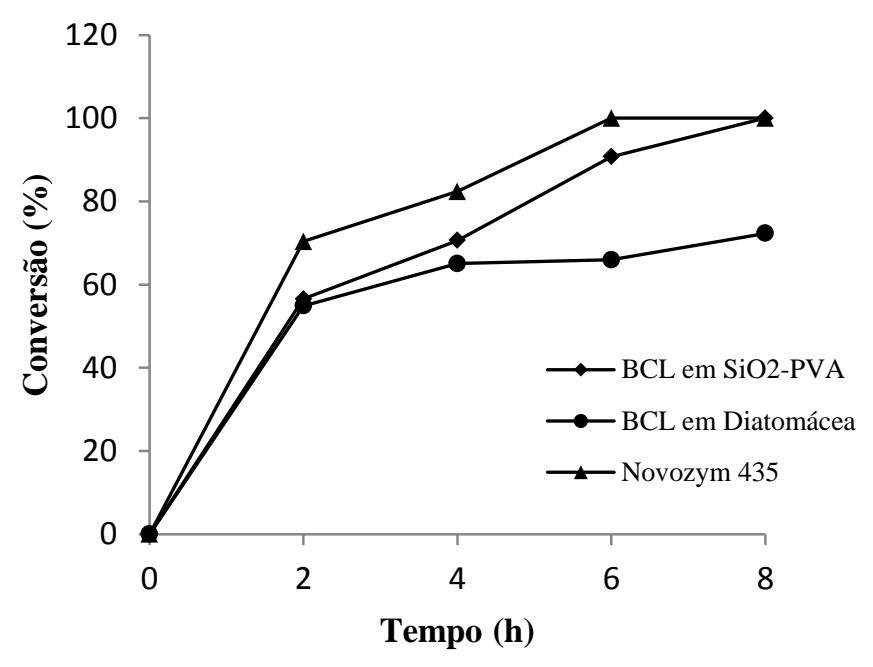

Figura 3 - Perfil da conversão em ésteres etílicos empregando ultrassom.

A redução do tempo de processamento de aquecimento convencional de $72 \mathrm{~h}$ para apenas $8 \mathrm{~h}$ foi observada quando utilizado o ultrassom como fonte de energia na transesterificação enzimática de óleo de coco bruto com B. cepacia imobilizada em $\mathrm{SiO}_{2}$-PVA, enquanto que para Novozym 435 foi observada a redução de 24h para 6h, como mostrado na Figura 4. De acordo com Kumar et al. (2011) na área da catálise enzimática, onde o catalisador se encontra numa fase diferente dos reagentes, a forma de dispersão ultrassônica aumenta a área de superfície disponível para os reagentes e, essencialmente, a cavitação aumenta a eficácia do catalisador para alcance da conversão ótima. 


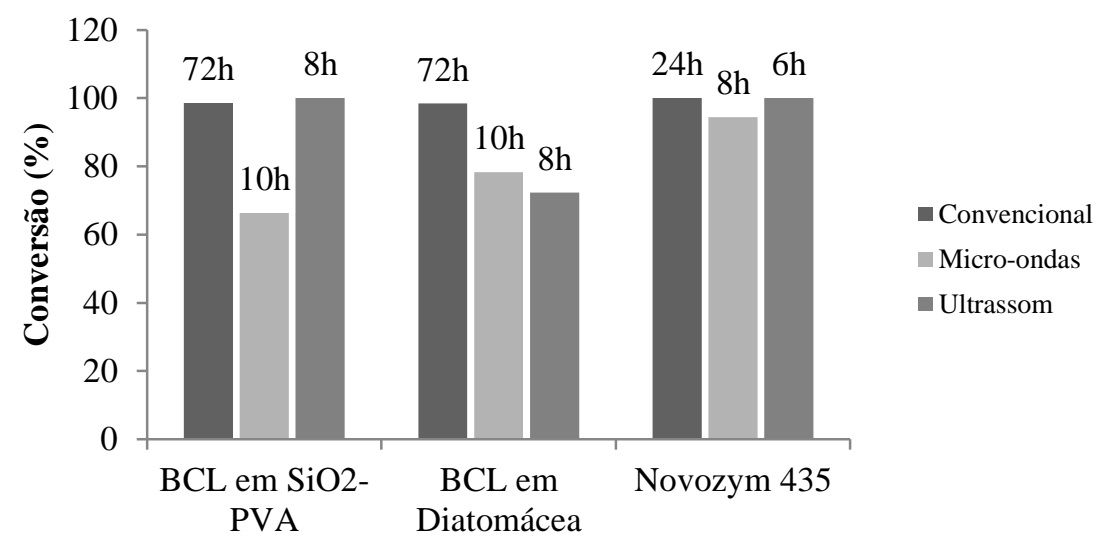

Figura 4 - Comparação das conversões alcançadas nas reações de transesterificação enzimática empregando diferentes derivados imobilizados sob diferentes sistemas de aquecimento em relação ao tempo.

\section{CONCLUSÃO}

Este estudo demonstrou que o meio reacional, quando submetido ao ultrassom promoveu uma redução no tempo de reação, em comparação com os outros sistemas de aquecimento: meio convencional e microondas. Por conseguinte, o óleo de coco bruto, apesar de apresentar características desfavoráveis, foi convertido em $100 \%$ de ésteres etílicos na reação que necessitou apenas de $8 \mathrm{~h}$ para o biocatalisador B. cepacia imobilizada em $\mathrm{SiO}_{2}$-PVA e $6 \mathrm{~h}$ para Novozym 435 enquanto que os processo empregando aquecimento convencional a conversão máxima ocorreu com $72 \mathrm{~h}$ para B. cepacia imobilizada em $\mathrm{SiO}_{2}-\mathrm{PVA}$ e $24 \mathrm{~h}$ para Novozym 435.

\section{REFERÊNCIAS}

AZÓCAR, L.; CIUDAD, G.; HEIPIEPER, H. J.; MUÑOZ, R.; NAVIA, R. Improving fatty acid methyl ester production yield in a lipase-catalyzed process using waste frying oils as feedstock. $J$. Biosci. Bioeng., v. 109, p. 609-614, 2010.

CHEN, K-S.; LIN, Y-C.; HSU, K-H.; WANG, H-K. Improving biodiesel yields from waste cooking oil by using sodium methoxide and a microwave heating system. Energy, v. 38, p. 151-156, 2012.

CINTAS, P.; MANTEGNA, S.; GAUDINO, E. C.; CRAVOTTO, G. A new pilot flow reactor for high-intensity ultrasound irradiation. Application to the synthesis of biodiesel. Ultrasonics Sonochem, v. 17, p. 985-989, 2010.

DA RÓS, P. C. M.; CASTRO, H. F.; CARVALHO, A. K. F.; SOARES, C. M. F.; MORAES, F. F.; ZANIN, G. M. Microwave-assisted enzymatic synthesis of beef tallow biodiesel. J. Ind. Microbiol. Biotechnol., v. 39, p. 529-536, 2012.

FREITAS, L.; DA RÓS, P. C. M.; SANTOS, J. C.; DE CASTRO, H. F. An integrated approach to produce biodiesel and monoglycerides by enzymatic interesterification of babassu oil (Orbinya sp). 
Process Biochem., v. 44, p. 1068-1074, 2009.

IBRAHIM, N. A.; NIELSEN, S. T.; WIGNEWARAN, V.; ZHANG, H.; XU, X. Online prepurification for the continuous enzymatic interestification of bulk fats containing omega-3 oil. J. Am. Oil Chem. Soc., v. 85, p. 95-98, 2007.

KANITKAR, A.; BALASUBRAMANIAN, S.; LIMA, M.; BOLDOR, D. A critical comparison of methyl and ethyl esters production from soybean and rice bran oil in the presence of microwaves. Bioresour. Technol, 2011.

KUMAR, D.; KUMAR, G.; POONAM, SINGH, C. P. Fast, easy ethanolysis of coconut oil for biodiesel production assisted by ultrasonication. Ultrason Sonochem., v. 17, p. 555-559, 2010.

NOUREDDINI, H.; GAO, X.; PHILKANA, R. S. Immobilized Pseudomonas cepacia lipase for biodiesel fuel production from soybean oil. Bioresour. Technol., v. 96, p. 769-777, 2005.

OLIVEIRA, J. F. G.; LUCENA, I. L.; SABOYA, R. M. A.; RODRIGUES, M. L.; TORRES, A. E. B.; FERNANDES, F. A. N.; CAVALCANTE JR., C. L.; PARENTE JR, E. J. S. Biodiesel production from waste coconut oil by esterification with ethanol: The effect of water removal by adsorption. Renew. Energ., v. 35, p. 2581-2584, 2010.

RIBEIRO, L. M. O.; SANTOS, B. C. S.; RENATA M.R.G. ALMEIDA, R. M. R. G. Studies on reaction parameters influence on ethanolic production of coconut oil biodiesel using immobilized lipase as a catalyst. Biomass Bioenerg., v. 47, p. 498-503, 2012.

SOARES, C. M. F.; CASTRO, H. F.; MORAES, F. F.; ZANIN, G. M. Characterization and utilization of Candida rugosa lipase immobilized on controlled pore silica. Appl. Biochem. Biotechnol., v. 77-9, p. 745-757, 1999.

STAMENKOVIC, O. S.; VELIC`KOVIC’, A. V.; VELJKOVIC, V. B. The production of biodiesel from vegetable oils by ethanolysis: Current state and perspectives. Fuel, v. 90, p. 3141-3155, 2011.

URIOSTE, D.; CASTRO M. B. A,; BIAGGIO F. C, DE CASTRO H. F. Synthesis of chromatographic standards and establishment of a method for the quantification of the fatty ester composition of biodiesel from babassu oil. Quim. Nova, v. 31, p. 407-412, 2008.

VELJKOVIC, V. B.; AVRAMOVIC, J. M.; STAMENKOVIC, O. S. Biodiesel production by ultrasound-assisted transesterification: State of the art and the perspectives. Renew. Sust. Energ. Rev., v. 16, p. 1193-1209, 2012. 